\title{
Análisis de las tasas de captura de jurel (Trachurus symmetricus) en la zona centro-sur de Chile (1987-1999)
}

\author{
Claudio Gatica y Luis Cubillos \\ Instituto de Investigación Pesquera, Casilla 350, Talcahuano, Chile \\ E-mail: cgatica@inpesca.cl
}

Recibido: 22 junio 2001; versión corregida: 4 noviembre 2002; aceptado: 15 noviembre 2002

\begin{abstract}
RESUMEN. Se analizaron los cambios en la Captura por Unidad de Esfuerzo (CPUE) de la pesquería industrial de cerco de jurel (Trachurus symmetricus) en la zona centro-sur de Chile entre 1987 y 1999. Se utilizaron dos unidades de esfuerzo de pesca para calcular las tasas de captura: días fuera de puerto y viajes con pesca. La flota se clasificó en cuatro estratos homogéneos mediante Análisis de Componentes Principales (ACP), utilizando una matriz de correlación de variables técnico-operacionales. Los dos primeros ejes del ACP explicaron 87,8\% de la varianza, debido a la naturaleza altamente autocorrelacionada de las variables. Los estratos identificados se utilizaron como variables categóricas para estandarizar la CPUE a través de un Modelo Lineal General (MLG), previa transformación logarítmica de la CPUE. Los modelos ajustados a los datos explicaron sobre un 64\% de la variabilidad observada, siendo el estrato el que explicó un mayor porcentaje de variabilidad: 24,6\% en el caso de la CPUE basada en los días fuera de puerto y 42,6\% en la CPUE basada en los viajes con pesca. Las dos series de CPUE indicaron diferentes tendencias entre 1987 y 1993, pero coincidieron en señalar una tendencia negativa de la CPUE entre 1994 y 1997. La clasificación por estratos de una flota heterogénea mediante ACP permitió mejorar el análisis de las tasas de captura, aunque la componente espacial debe ser tomada en cuenta en futuros estudios. A su vez, la unidad de esfuerzo de pesca puede tener un impacto notable en las tendencias de CPUE, particularmente si se considera como un índice de abundancia relativa.
\end{abstract}

Palabras claves: ACP, CPUE, MLG, jurel, Trachurus symmetricus.

\section{Jack mackerel (Trachurus symmetricus) catch rate analysis in the central-south of Chile (1987-1999)}

\begin{abstract}
Catch per unit effort (CPUE) data from the jack mackerel fishery operating in central-south Chile and covering the period 1987-1999 are analyzed in order to detect changes in relative abundance. Two units for fishing effort were used to compute the catch rate: number of days outside port and the number of trips with capture. The fleet was classified in four homogeneous strata by using a principal component analysis (PCA) based on the correlation matrix of technical-operational variables of the purse-seine fleet. The first component explained $87.8 \%$ of the variation because the highly correlationed variables. The four homogeneous strata were used as categorical variables to standardize CPUE through a General Linear Model (MLG), previous log-transformation of CPUE data. The first model, based on catch per days outside port, explained more than $64 \%$ of the observed variability. In this model, the stratum explains an important fraction of the total variability (24.6\%). Similarly, in the case of the CPUE based on trips with capture, a model explained $(42.6 \%)$ of the total variability and particularly the stratum. However, the two time series of CPUE indicate different tendencies in the annual coefficients for the 1987-1993 period. From 1994 to 1997, the two series exhibit a negative tendency in the annual coefficients. The analysis of catch rate can be improved when a multivariate approach is applied to a heterogeneous fleet, but spatial component should be take into account in future studies. Furthermore, the fishing effort unit can impact remarkably the tendencies of standardized CPUE, particularly if this is considered as a relative index of abundance.
\end{abstract}

Key words: ACP, CPUE, MLG, jack mackerel, Trachurus symmetricus. 


\section{INTRODUCCIÓN}

El jurel (Trachurus symmetricus) es una especie pelágica altamente migratoria, que presenta una amplia distribución geográfica en el Pacífico sur. Esta cubre desde las Islas Galápagos hasta la región austral de Chile, tanto en aguas oceánicas como costeras (Serra, 1991). En aguas internacionales se distribuye en grandes cardúmenes hasta $160^{\circ} \mathrm{W}$, y principalmente entre los $33^{\circ} \mathrm{S}$ y $48^{\circ} \mathrm{S}$ (Grechina, 1992).

Hasta 1991, el jurel fue explotado por la flota industrial de cerco chilena, así como por una flota industrial de altura de origen ruso, que operó entre 1978 y 1991 fuera de las $200 \mathrm{mn}$ frente a Chile (Serra, 1991). Actualmente, sólo opera la flota chilena siendo la zona de operación centro-sur la más importante en términos de rendimientos de pesca. Desde 1997 la pesquería se encuentra en un período de sucesivas vedas y restricciones, debido a una juvenilización de las capturas (SUBPESCA, 1999).

En la evaluación, seguimiento y análisis de la dinámica del jurel se han utilizado datos de captura y esfuerzo obtenidos de la actividad de la flota industrial de cerco (Böhm et al., 1997; Serra et al., 1998b, 1998b). Las tasas de captura o captura por unidad de esfuerzo (CPUE) han sido analizadas como indicadores de abundancia relativa del stock, para ser incorporados en la calibración de modelos de evaluación de stock edad-estructurados como Análisis Secuencial de la Población (Serra et al., 1998a). Aunque se ha intentado corregir estos índices para aislar el efecto producido por cambios en la composición de la flota a medida que ésta se desarrolla (Böhm et al., 1997), de acuerdo con Allen y Punsly (1984) y Sainsbury et al., (1999), varios otros factores como el efecto de variables ambientales, que pueden afectar la disponibilidad del recurso (Hinton y Nakano, 1996), no han sido aún tomados en cuenta. Sin embargo, uno de los aspectos muchas veces subestimado dice relación con la elección de una unidad de esfuerzo apropiada para calcular las tasas de captura, como también el clasificar adecuadamente las embarcaciones de una flota heterogénea en grupos relativamente homogéneos.

Para estandarizar las tasas de captura existen dos enfoques principales. El primero está basado en los trabajos de Shimada y Shaeffer (1956), y considera un enfoque de diseño experimental, donde todas las embarcaciones que actúan en un área determinada son comparadas con una embarcación estándar. Otro enfoque utiliza Modelos Lineales
Generalizados (MLG) para estandarizar las tasas de captura. En este tipo de modelos es posible incorporar tanto variables físicas como categóricas asociadas con la evolución de la flota y que pueden resultar en cambios de capturabilidad (Quinn y Deriso, 1999). Además, los MLG son flexibles a diferentes formas de analizar la información, en especial sobre el ensayo de diferentes alternativas de distribución del error, utilizado en el ajuste por medio del principio de máxima verosimilitud (Mc Cullagh y Nelder, 1989). Las características de los MLG han preferido este enfoque, siendo en la actualidad el más usado en la corrección de las tasas de captura (Yáñez et al., 1999; Punt et al., 2000; Simpfendorfer et al., 2000). Este método de estandarización requiere del análisis de las distintas variables que pueden afectar la CPUE (Hinton y Nakano, 1996), siendo generalmente incorporado el estrato como una variable categórica en la modelación (Kimura, 1981; Goñi et al., 1999). En este contexto, la caracterización por estratos se realiza considerando sólo alguna característica técnico-operacional de las embarcaciones. Sin embargo, la eficiencia de una embarcación no está determinada por un solo factor (Kimura, 1981). Por lo tanto, la caracterización de la flota en estratos por medio de un análisis multivariado puede fortalecer la estandarización de las tasas de captura, ya que la variabilidad en el esfuerzo de pesca es incorporada por la combinación de un conjunto de variables y la mantención de la varianza total luego de sucesivas transformaciones (Hernández y Sepúlveda, 1998).

En este trabajo se aplica un Análisis de Componentes Principales (ACP), para caracterizar la flota de una pesquería altamente heterogénea como la flota industrial de cerco del jurel, en estratos homogéneos de embarcaciones. Posteriormente, el estrato se incorpora como una variable categórica en la modelación de la CPUE, para evaluar su efecto y obtener una serie de CPUE estandarizada. Con el objeto de analizar el efecto de la unidad de esfuerzo en las tasas de captura, se construyeron dos series de tasas de captura. Una consideró los viajes con pesca de jurel y la otra, los días fuera de puerto utilizados en la captura del recurso. La primera unidad de esfuerzo fue utilizada por Böhm et al., (1997), ya que presenta una alta correlación con las capturas. En este sentido, los viajes con pesca pueden constituirse en una unidad que no refleje el desarrollo no exitoso de un viaje. Por otra parte, los días fuera de puerto pueden ser interpretados como mayor tiempo requerido en la búsqueda de agregaciones 
comerciales, y podrían reflejar de mejor forma los viajes sin éxito de una embarcación.

\section{MATERIALES Y MÉTODOS}

\section{Área de estudio}

El área de estudio correspondió a la zona centro-sur de Chile, y comprendió el área de operación de la flota cerquera industrial en la pesquería del jurel que opera principalmente entre los $35^{\circ}$ y $40^{\circ} \mathrm{S}$ (Fig. 1).

\section{Base de datos}

La información analizada proviene de registros operacionales de las embarcaciones industriales de cerco que operaron entre 1987 y 1999. Esta información fue generada por el programa de estadística pesquera y el programa sinóptico de pesca del Instituto de Investigación Pesquera. La información tecnológica correspondió a características técnicooperacionales de las embarcaciones, que fueron: eslora (longitud del barco), manga (ancho del barco), puntal (altura entre dos cubiertas), capacidad de bodega (volumen en $\mathrm{m}^{3}$ para carga), potencia

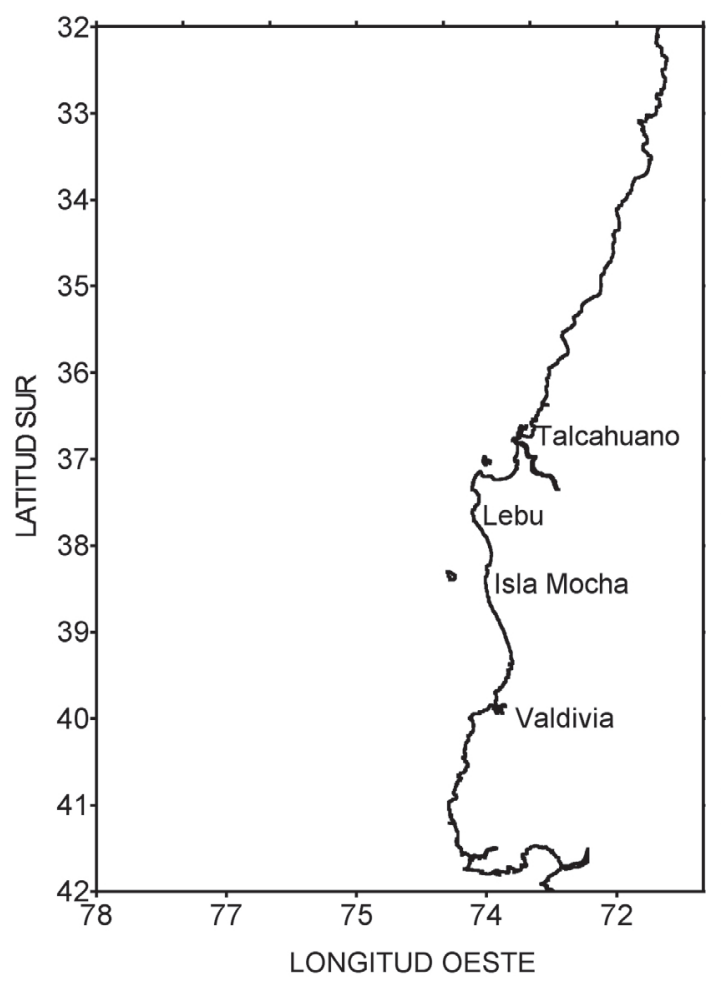

Figura 1. Área de operación en la pesquería del jurel. Figure 1. Operation area in jack mackerel fishery. motor principal (HP del motor), TRG (tonelaje de registro grueso) y LRED (largo de red), mientras que la información operacional fueron los registros diarios de captura (toneladas) y de esfuerzo de pesca.

\section{Unidad de esfuerzo de pesca}

En este estudio se consideraron como medidas de esfuerzo nominal los viajes con captura de jurel (vcp) y los días fuera puerto (dfp) utilizados en esta captura. Los viajes con pesca totales no se utilizaron porque solo se analizó las tasas de captura asociadas directamente a la especie objetivo. Además, los registros con capturas nulas no estaban completos lo cual dificultó la utilización de los viajes totales como unidad de esfuerzo en la modelación, siendo ésta la mayor razón para no considerarlos.

\section{Determinación de estratos}

Para la determinación de estratos de embarcaciones homogéneos se aplicó un Análisis de Componentes Principales (ACP), el cual usualmente se utiliza en la identificación de unidades discretas homogéneas (Pease, 1999). En este trabajo, producto de la diversidad de medidas de las variables físicas cuantificables de la flota, el ACP se realizó sobre una matriz de correlación (Hernández y Sepúlveda, 1998), siendo obtenidos los puntajes asociados a cada componente principal. Para este objetivo las variables fueron previamente transformadas por uso de logaritmo natural, para reducir la varianza de los datos y homogenizar sus unidades. Las seis variables técnico-operacionales (tonelaje de registro grueso, largo de red, potencia de la embarcación, velocidad máxima, eslora y manga), se analizaron en relación a la CPUE obtenida por cada embarcación considerando como unidad de esfuerzo los días fuera de puerto de cada embarcación entre 1987 y 1999.

La base de datos permitió caracterizar sobre un $50 \%$ de la flota que operó en la pesquería del jurel entre 1987 y 1999. En consecuencia, en el análisis de tasas de captura sólo se utilizaron los datos de captura y esfuerzo de las embarcaciones clasificadas en un determinado estrato, que al superar en promedio el $50 \%$ de las embarcaciones, puede considerarse representativo de la composición de la flota.

\section{Estandarización de la CPUE con MLG}

La utilización de la CPUE como índice de abundancia relativa está basado en el supuesto de proporcionalidad entre la CPUE y la abundancia pro- 
medio anual $\overline{\mathrm{N}}_{\mathrm{i}}$ obtenida por una clase de embarcación discreta (Kimura, 1981). Esta relación es más evidente al analizar la siguiente ecuación de captura.

$$
\mathrm{C}_{\mathrm{ij}}=\mathrm{q} * \mathrm{f}_{\mathrm{ij}} * \overline{\mathrm{N}}_{\mathrm{i}}
$$

donde $\mathrm{C}_{\mathrm{ij}}$ es la captura en peso, $\mathrm{f}_{\mathrm{ij}}$ es el esfuerzo en las unidades correspondientes (generalmente representado por el tiempo dedicado a la captura, número de barcos operando, número de viajes u otra medida) y q es el coeficiente de capturabilidad (Hilborn y Walters, 1992).

La metodología de estandarización corresponde al enfoque a través de Modelos Lineales Generalizados (Hilborn y Walters, 1992), y basado en los trabajos de Kimura (1981), y Allen y Punsly (1984). Al definir la captura por unidad de esfuerzo (CPUE) en el año i del barco j como el estimador de razón:

$$
U_{i, j}=\frac{\sum_{m=1}^{M} C_{i, j, m}}{\sum_{m=1}^{M} f_{i, j, m}}
$$

Con $\mathrm{U}_{\mathrm{i}, \mathrm{j}}$ como la CPUE, $\mathrm{m}$ el índice del viaje de pesca y $\mathrm{M}$ el número total de viajes realizados por la embarcación en un año determinado y suponiendo que $\mathrm{U}_{\mathrm{i}, \mathrm{j}}$ como índice de abundancia relativa es el producto de los efectos multiplicativos de la abundancia en el mar y de propiedades categóricas y cuantitativas de las embarcaciones, así como de variables de tipo ambiental se tiene que:

$$
\mathrm{U}_{\mathrm{i}, \mathrm{j}}=\alpha_{\mathrm{i}}\left(\mathrm{Q}_{\mathrm{ij}}\right)
$$

Donde $\alpha_{\mathrm{i}}$ representa el efecto de la variación anual de la abundancia en el mar, y $\mathrm{Q}_{\mathrm{ij}}$ es el conjunto de efectos derivados de las características o decisiones de pesca de cada barco en conjunto con variables de tipo ambiental. Luego, como el objetivo es la construcción de una serie de abundancia relativa, es necesario despejar el coeficiente $\alpha$ a través de los años. Para obtener estos coeficientes es necesario modelar $\mathrm{U}_{\mathrm{i}, \mathrm{j}}$, para ello se utilizó un modelo multiplicativo que explica la relación entre la CPUE y las distintas variables que la afectan. En esta investigación el modelo a construir correspondió a:

$$
\mathrm{U}_{\mathrm{i}, \mathrm{j}, \mathrm{h}, \mathrm{r}}(\mathrm{k})=\alpha_{\mathrm{i}} \mathrm{b}_{\mathrm{j}} \mathrm{e}_{\mathrm{h}} \mathrm{z}_{\mathrm{r}} \mathrm{k}^{\beta}
$$

donde $b_{\mathrm{j}}$ es el efecto del barco, $e_{h}$ es el efecto del estrato, $\mathrm{z}_{\mathrm{r}}$ es el efecto de la estación y $\mathrm{k}^{\beta}$ es la capacidad de bodega. La serie completa correspondió a una combinación barco-año-estación-estrato-capacidad de bodega que en forma arbitraria se fijó en un estrato representativo de la flota, que en esta investigación correspondió al estrato 3 en el primer año de la serie y primera estación. La variable estación se clasificó en 4 tipos: a) Estación 1 (diciembre-febrero), b) Estación 2 (marzo-mayo), c) Estación 3 (junio-agosto) y d) Estación 4 (septiembrenoviembre). De esta forma se obtuvo un modelo estadístico multiplicativo perteneciente a la familia de MLG (Mc Cullagh y Nelder, 1989).

$$
{ }_{\mathrm{f}} \mathrm{U}_{\mathrm{i}, \mathrm{j}, \mathrm{h}, \mathrm{r}}(\mathrm{k})=\alpha_{\mathrm{i}} \mathrm{b}_{\mathrm{j}} \mathrm{e}_{\mathrm{h}} \mathrm{Z}_{\mathrm{r}} \mathrm{k}^{\beta} \varepsilon_{\mathrm{i}, \mathrm{j}, \mathrm{h}, \mathrm{r}}(\mathrm{k})
$$

donde $\varepsilon$ es un error aleatorio de distribución lognormal. La versión logarítmica de este modelo multiplicativo generó un modelo aditivo de la forma:

$$
\begin{gathered}
\log \left(\mathrm{f}_{\mathrm{i}, \mathrm{j}, \mathrm{h}, \mathrm{r}}(\mathrm{k})\right)=\log \left(\mathrm{U}_{1,1,3,1}\right)+ \\
+\log \left(\alpha_{\mathrm{i}}\right)+\log \left(\mathrm{b}_{\mathrm{j}}\right)+\log \left(\mathrm{e}_{\mathrm{h}}\right)+ \\
+\log \left(\mathrm{z}_{\mathrm{r}}\right)+\beta \log (\mathrm{k}) \log (\varepsilon)
\end{gathered}
$$

En este modelo mediante un ANOVA y la partición de sus componentes se puede obtener los estimadores de $\log \left(\alpha_{\mathrm{i}}\right)$ de cada año. Es necesario considerar que la transformación logarítmica generalmente produce que el error de distribución lognormal asuma una distribución normal o muy cercana a la normal (Sainsbury et al., 1999).

El ajuste del MLG es realizado con el procedimiento paso a paso de selección descrito por Allen y Punsly (1984). Este método consiste en ajustar un modelo inicial con todas las variables e interacciones posibles. Posteriormente, se seleccionan sólo las variables altamente significativas $(\mathrm{P}<0,001)$, eliminando del modelo aquellas variables e interacciones poco significativas. Este proceso debe ser riguroso, ya que debido a la gran cantidad de observaciones en las series de datos, se puede producir una parametrización excesiva, capaz de diluir otros efectos (Punsly y Deriso, 1991).

El proceso de estimación de los parámetros en los MLG está basado en el principio de máxima ve- 
rosimilitud, dependiente de la distribución del error (Mc Cullagh y Nelder, 1989; Chambers y Hastie, 1993). Estos modelos permiten ensayar distintas alternativas de distribución para el error (Goñi et al., 1999; Punt et al., 2000), siendo finalmente la selección del modelo para el error dependiente de la distribución de los datos.

El ajuste del modelo se realizó utilizando las rutinas del programa S-plus (Chambers y Hastie, 1993), obteniéndose los coeficientes del MLG para cada variable incluida en la modelación de la CPUE. Luego, como se tiene la totalidad de los efectos de cada variable sobre la CPUE, se puede despejar el efecto de cada uno y encontrar el término asociado a la abundancia relativa presente en la naturaleza $\left(\alpha_{i}\right)$ para cada año de la serie. Como estos coeficientes se encuentran en unidades de logaritmo, se realizó una retro-transformación exponencial de los estimadores para que la serie de $\left(\alpha_{i}\right)$ se trabaje en unidades naturales. Estos coeficientes de $\left(\alpha_{\mathrm{i}}\right)$ corresponden a promedios estandarizados de abundancia relativa anual, los cuales son corregidos a causa del sesgo producido con la transformación logarítmica (Kimura, 1981), por medio de la siguiente expresión:

$$
\alpha_{i}=e^{\log \left(\alpha_{t}\right)} e^{\frac{-\sigma^{2}}{2}}
$$

Este índice resulta en la serie de abundancia relativa estandarizada que puede ser utilizada para la ca- libración de modelos de evaluación. La serie de CPUE estandarizada es comparable con la serie de CPUE nominal, ya que ambas entregan las tendencias anuales en promedio relativas a un estrato característico de la flota cerquera del jurel. Aunque es posible trabajar escalas de tiempo distintas a la anual, esto no se justifica hacia una serie de CPUE estandarizada que tiene por objetivo ser utilizada como calibrador de modelos, edad o tallaestructurados e indicador de la abundancia relativa del recurso.

\section{RESULTADOS}

\section{Análisis de Componentes Principales (ACP)}

Los resultados obtenidos indican la existencia de cuatro estratos homogéneos de embarcaciones (Fig. 2), obtenidos por la separación de los puntajes a lo largo de la primera componente principal. La combinación de variables que entregó la mejor segregación de estratos incluyó TRG (tonelaje de registro grueso), LRED (largo de red), HP (potencia de la embarcación), VMAX (velocidad máxima), ESL (eslora) y MGA (manga). Estos estratos son homogéneos ya que constituyen agrupaciones naturales producto del grado de asociación entre las variables, evidenciada sobre una matriz de correlación. Los estratos presentaron una sobreposición en el rango de variables, que se explicó por la naturaleza multivariada del análisis que permite que los gru-

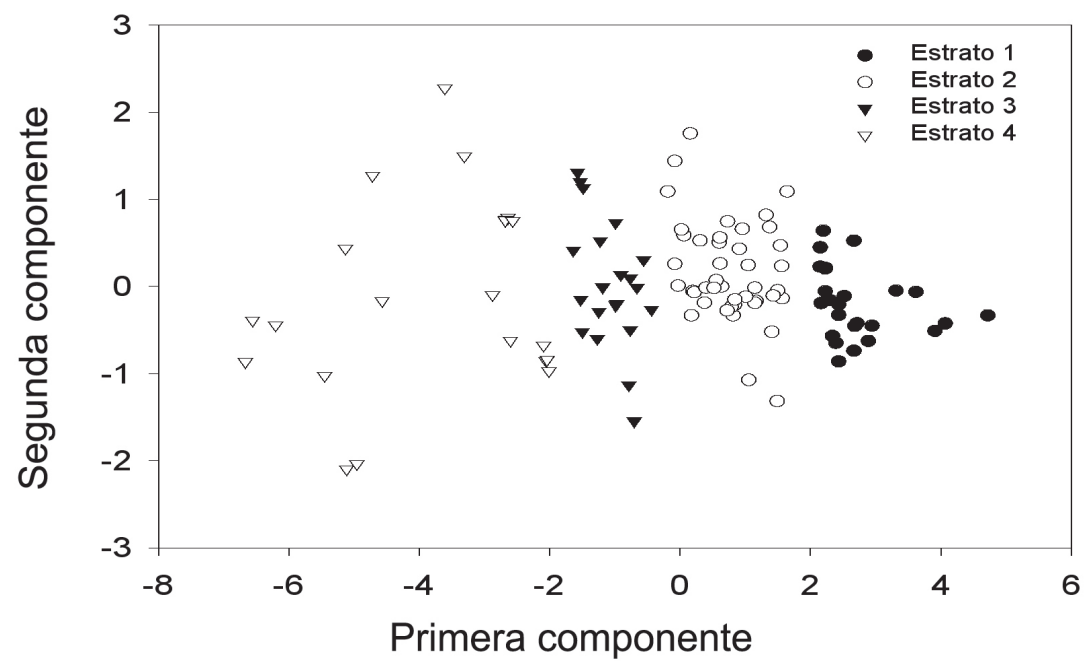

Figura 2. Puntajes del ACP sobre la primera y segunda componente principal.

Figure 2. First and second main components of PCA. 
pos existan como una combinación entre variables y sus correspondientes rangos. El resultado del ACP implementado con estas variables indicó que la primera componente principal explicó el 80,6\% de la varianza total de los datos (Tabla 1).

\section{Tasas de captura estandarizadas}

Los resultados presentados corresponden a los mejores ajustes y modelos obtenidos en base a distintas combinaciones de variables e interacciones. $\mathrm{Si}$ bien existe la posibilidad de otras interacciones en el modelo, éstas no fueron significativas o su aporte para explicar la variable respuesta de interés fue bajo en el incremento de $\mathrm{R}^{2}$.

\section{Serie CPUE (ton/dfp)}

En esta serie el modelo de distribución del error seleccionado correspondió a uno de distribución normal.

$$
\begin{aligned}
\mathrm{Y}_{\mathrm{i}, \mathrm{j}, \mathrm{k}} & =\mu+\mathrm{A}_{\mathrm{i}}+\mathrm{E}_{\mathrm{j}}+\mathrm{S}_{\mathrm{k}}+\mathrm{B}_{\mathrm{h}}+\mathrm{AS}_{\mathrm{i}, \mathrm{k}}+\varepsilon_{\mathrm{i}, \mathrm{j}, \mathrm{k}} \\
\mathrm{Y}_{\mathrm{i}, \mathrm{j}, \mathrm{k}} & =\text { logaritmo de la CPUE (ton/dfp) } \\
\mu & =\text { promedio general } \\
\mathrm{A}_{\mathrm{i}} & =\text { efecto del año i } \\
\mathrm{E}_{\mathrm{j}} & =\text { efecto de la estación } \mathrm{j} \\
\mathrm{S}_{\mathrm{k}} & =\text { efecto del estrato } \mathrm{k} \\
\mathrm{B}_{\mathrm{h}} & =\text { efecto de capacidad bodega } \mathrm{h} \\
\mathrm{AS}_{\mathrm{i}, \mathrm{k}} & =\text { efecto de la interacción entre } \mathrm{A}_{\mathrm{i}} \mathrm{y} \mathrm{S}_{\mathrm{k}} \\
\varepsilon_{\mathrm{i}, \mathrm{j}, \mathrm{k}} & =\text { error }
\end{aligned}
$$

El ANOVA presentó efectos significativos para el año, estación, estrato, capacidad de bodega e interacción año-estrato, indicando que la CPUE cambia de año en año, de estación en estación y a través de los estratos (Tabla 2). La interacción mostró que la CPUE cambió de acuerdo a una combinación entre año y estrato. El modelo completo explicó un porcentaje significativo de la variación del log CPUE $\left(R^{2}=0,64\right)$, siendo significativo el efecto del estrato, explicando por sí solo el $24,6 \%$ de la variabilidad del log CPUE.

En el periodo 1987-1994, la serie estandarizada presentó una tendencia negativa de la CPUE, mientras que la serie nominal mostró una tendencia positiva hasta 1994 (Fig. 3). Luego, de 1994 a 1998 ambas series indicaron una tendencia negativa, que cambió a positivo desde 1998 a 1999. La comparación entre las tendencias nominales y estandarizadas es posible, ya que ambas series corresponden a promedios y el origen de la serie estandarizada desde un MLG, es consecuencia de la modelación y no es inconsistente usar estas series en términos comparativos de tendencia.

Para validar el modelo se realizaron pruebas de diagnóstico que incluyeron el análisis de los residuos y gráficos de los valores observados y predichos por el modelo. En la Figura 4 se observó una buena relación entre los valores predichos y observados, con una baja dispersión de las observaciones. En forma complementaria, el análisis de los residuos contra los valores predichos no indicó ningún patrón sistemático que haga sospechar la falta de independencia que invalide los resultados del modelo (Fig. 5). Finalmente, el histograma de los residuos mostró que no existen problemas con la normalidad (Fig. 6), siendo el modelo considerado estadísticamente válido.

\section{Serie CPUE(ton/vep)}

En esta serie, el modelo ajustado presentó efectos significativos de año, estación, estrato, capacidad de bodega e interacción año-estrato, siendo utilizado un modelo de distribución normal para el error:

$$
\begin{aligned}
\mathrm{Y}_{\mathrm{i}, \mathrm{j}, \mathrm{k}} & =\mu+\mathrm{A}_{\mathrm{i}}+\mathrm{E}_{\mathrm{j}}+\mathrm{S}_{\mathrm{k}}+\mathrm{B}_{\mathrm{h}}+\mathrm{AS}_{\mathrm{i}, \mathrm{k}}+\varepsilon_{\mathrm{i}, \mathrm{j}, \mathrm{k}} \\
\mathrm{Y}_{\mathrm{i}, \mathrm{j}, \mathrm{k}} & =\text { logaritmo de la CPUE (ton/dfp) } \\
\mu & =\text { promedio general } \\
\mathrm{A}_{\mathrm{i}} & =\text { efecto del año i } \\
\mathrm{E}_{\mathrm{j}} & =\text { efecto de la estación } \mathrm{j} \\
\mathrm{S}_{\mathrm{k}} & =\text { efecto del estrato } \mathrm{k} \\
\mathrm{B}_{\mathrm{h}} & =\text { efecto de capacidad bodega } \mathrm{h} \\
\mathrm{AS}_{\mathrm{i}, \mathrm{k}} & =\text { efecto de la interacción entre } \mathrm{A}_{\mathrm{i}} \mathrm{y} \mathrm{S}_{\mathrm{k}} \\
\varepsilon_{\mathrm{i}, \mathrm{j}, \mathrm{k}} & =\text { error }
\end{aligned}
$$

El modelo ajustado explicó un $71 \%$ de variación del $\log$ CPUE $\left(\mathrm{R}^{2}=0,71\right)$, explicando el efecto del estrato un $42,7 \%$ del log CPUE. Estos resultados indicaron que la CPUE cambió de año en año, estación a estación, por estrato y de acuerdo a una combinación año-estrato (Tabla 4). Los valores de los coeficientes para el efecto año (Tabla 5), se utilizaron para analizar los cambios en tendencia de la CPUE, que mostraron una tendencia positiva entre 1987 y 1994. Sin embargo, desde 1994 se observó una señal negativa que cambió a positiva desde 1997 a 1999. En términos generales ambas series mostraron tendencias similares. No obstante, la serie de CPUE nominal entre 1995 y 1996 indicó una tendencia positiva. Finalmente, ambas series coincidieron en señalar la misma tendencia negativa hacia 1997, sugiriendo un cambio positivo en los últimos 
Tabla 1. Componentes de carga y porcentaje de varianza explicada por el ACP, donde: C (componente principal), CPUE (captura por unidad de esfuerzo), LRED (largo de red), HP (potencia del motor), VMAX (velocidad máxima), MGA (manga), ESL (eslora), TRG (tonelaje registro grueso), PV (porcentaje de varianza explicada) y PA (porcentaje acumulado).

Table 1. Load components and variance percentage explained by the PCA, where: C (main component), CPUE (catch per unit effort), LRED (size of the net), HP (horse power), VMAX (maximum speed), MGA (sleeve), ESL (length), TRG (gross tonnage registration), PV (percentage of explained variance) and PA (accumulated percentage).

\begin{tabular}{|ccccc|}
\hline Variable & C 1 & C 2 & C 3 & C 4 \\
\hline CPUE & 0,361 & 0,000 & $-0,730$ & $-0,452$ \\
ESL & 0,386 & 0,253 & 0,192 & 0,434 \\
HP & 0,400 & 0,000 & $-0,128$ & 0,338 \\
LRED & 0,372 & 0,117 & 0,621 & $-0,623$ \\
MGA & 0,390 & 0,000 & 0,111 & 0,000 \\
TRG & 0,407 & 0,171 & 0,000 & 0,310 \\
VMAX & 0,322 & $-0,882$ & $-0,124$ & 0,000 \\
PV & 0,806 & 0,072 & 0,044 & 0,031 \\
PA & 0,806 & 0,878 & 0,923 & 0,953 \\
\hline
\end{tabular}

Tabla 2. ANOVA del MLG ajustado a los datos de CPUE (días fuera de puerto).

Table 2. ANOVA of the adjusted MLG to the data of CPUE (days out of port).

\begin{tabular}{|cccccc|}
\hline Efecto & g.l. & $\begin{array}{c}\text { Suma } \\
\text { cuadrado }\end{array}$ & $\begin{array}{c}\text { Cuadrado } \\
\text { medio }\end{array}$ & $\begin{array}{c}\text { Valor } \\
\text { F }\end{array}$ & $\begin{array}{c}\text { Valor } \\
\mathbf{P}\end{array}$ \\
\hline Año & 12 & 11,98 & 1,00 & 24,41 & 0,00 \\
Estación & 3 & 97,35 & 32,45 & 793,53 & 0,00 \\
Estrato & 3 & 111,89 & 37,30 & 912,11 & 0,00 \\
$\begin{array}{c}\text { Capacidad } \\
\text { bodega }\end{array}$ & 1 & 7,18 & 7,18 & 175,66 & 0,00 \\
$\begin{array}{c}\text { Año: } \\
\text { Estrato }\end{array}$ & 36 & 6,38 & 0,18 & 4,34 & 0,00 \\
Residuos & 3172 & 129,71 & 0,04 & & \\
\hline
\end{tabular}

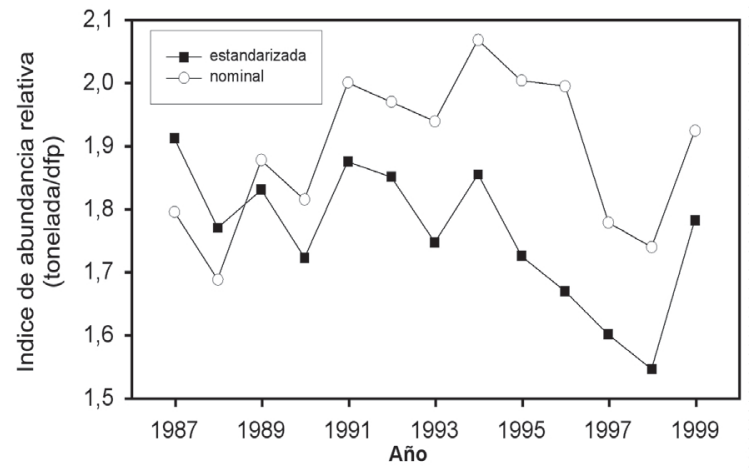

Figura 3. Tasa de captura (ton/dfp) estandarizada y nominal entre 1987 y 1999.

Figure 3. Catch rate (ton/dfp) standardized and nominal between 1987 and 1999.

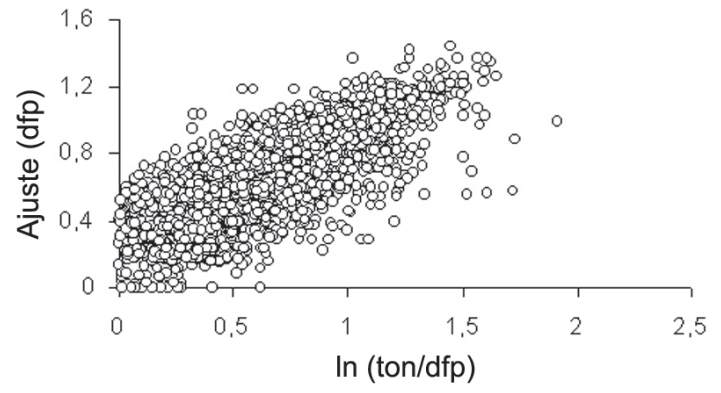

Figura 4. Valores observados (In (ton/dfp)) y ajustados por el MLG.

Figure 4. Observed and fitted values (ln (ton/dfp)) by MLG analysis.

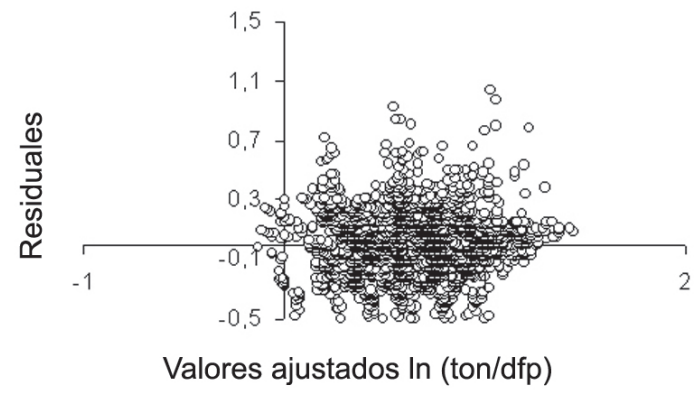

Figura 5. Residuales en función de los valores estimados (ln (ton/dfp)).

Figure 5. Residuals as a function of estimated values $(\ln ($ ton/dfp)). 


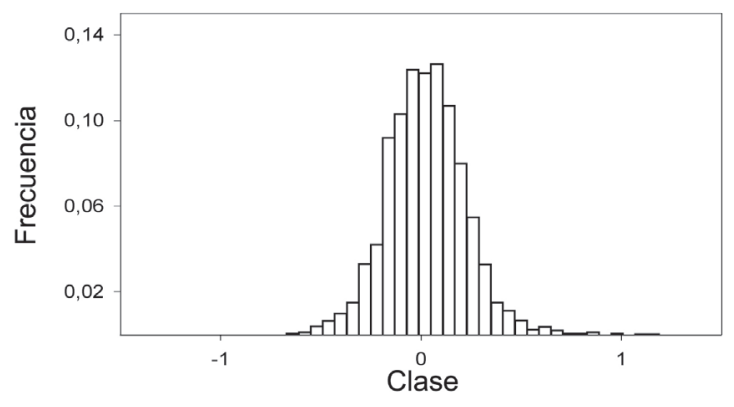

Figura 6. Histograma de los residuos del modelo ajustados a la serie CPUE (ton/dfp).

Figure 6. Histogram of residuals for the model fitted to CPUE (ton/dfp).

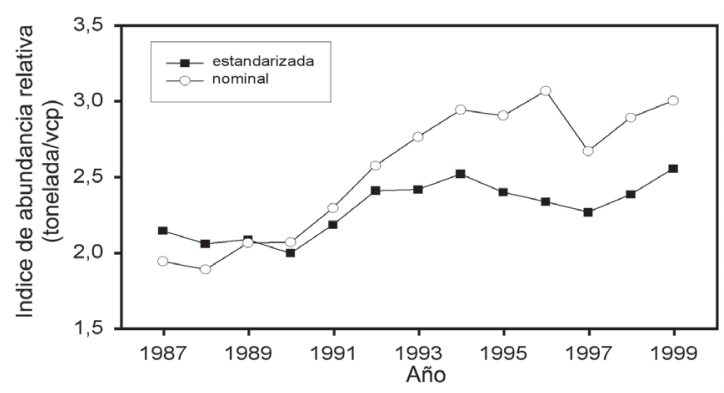

Figura 7. Tasa de captura (ton/vcp) estandarizada y nominal entre 1987 y 1999.

Figure 7. Catch rate (ton/vep) standardized and nominal between 1987 and 1999.

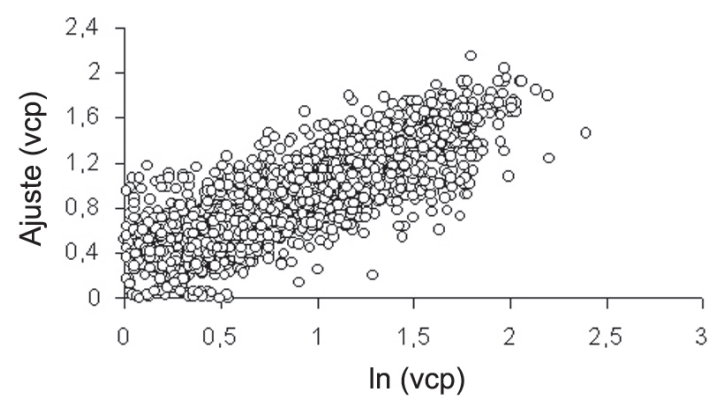

Figura 8. Gráfica de valores observados ln (vep) contra los ajustados por el MLG.

Figure 8. Graph of observed values ln (vep) against the fittings for the MLG. años de la serie, entre 1997 y 1999 (Fig. 7).

Los análisis de residuos realizados para verificar la validez de los supuestos del modelo incluyeron análisis de independencia, normalidad y homogeneidad de la varianza. El análisis gráfico entre los valores observados y los predichos por el modelo indicó un buen ajuste de los datos por el MLG (Fig. 8). El gráfico de los valores predichos contra los residuales no mostró ningún patrón sistemático asociado a la falta de independencia de las observaciones (Fig. 9). Luego, el histograma de los residuos mostró normalidad en las observaciones (Fig. 10). Por lo tanto, sobre la base de los análisis realizados se puede afirmar que el modelo es válido y que los coeficientes estandarizados son representativos como índices de abundancia del recurso.

\section{DISCUSIÓN}

La caracterización de la flota por medio del análisis multivariado es útil en el análisis de las tasas de captura de una flota heterogénea como la presentada en la pesquería de jurel. El ACP permite una buena caracterización a lo largo de la primera componente principal, y es el criterio de clasificación utilizado porque esta componente explicó la mayor variabilidad entre la variable de interés correspondiente a la CPUE (ton/dfp) y las variables técnicooperacionales de la flota. El efecto de la adecuada clasificación técnico-operacional de las embarcaciones se demostró a través de los altos valores del coeficiente de determinación $\left(\mathrm{R}^{2}\right)$ obtenidos al analizar los efectos principales en ambas series de CPUE y del porcentaje de suma de cuadrados que explicó cada variable. Lo señalado es avalado por el porcentaje de variación explicado por el efecto del estrato, con un $24,6 \%$ en la serie de CPUE (ton/dfp) y 42,7\% CPUE (ton/vcp), siendo este efecto el que explicó un mayor porcentaje de variabilidad en ambos modelos (Tablas 2 y 4 ).

Los MLG identificados evidenciaron la existencia de interacciones entre el año y estrato, indicando que la abundancia relativa del jurel podría presentar distintas tendencias en cada estrato, lo cual haría aconsejable su análisis por separado (Kimura y Zenger, 1997). No obstante, como estas interacciones explicaron sólo una pequeña fracción de la variación de los datos (Tablas 2 y 4), no tendrían un efecto muy importante sobre las CPUE estimadas. Luego, como ambos modelos son estadísticamente válidos, su interpretación debe ser 


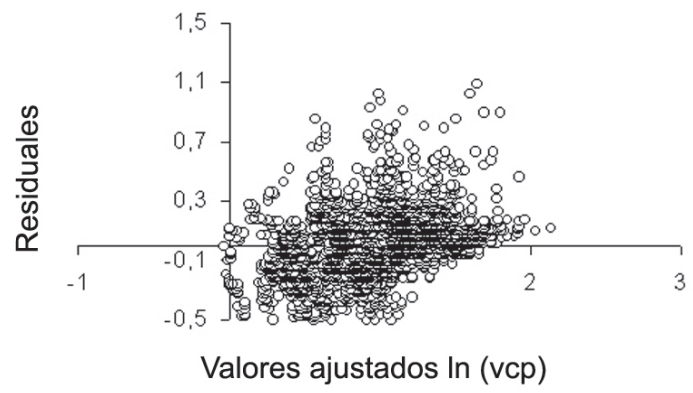

Figura 9. Valores ajustados $\ln (\mathrm{vcp})$ contra residuales. Figure 9. Adjusted values ln (vcp) against residual.

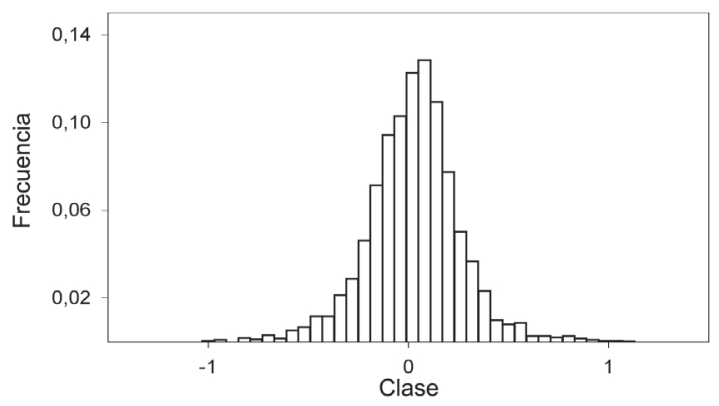

Figura 10. Histograma de los residuos del modelo ajustados a la serie CPUE (ton/vep).

Figure 10. Histogram of residuals of the adjusted model to the series CPUE (ton/vep).

Tabla 3. Coeficientes del efecto año obtenidos del modelo lineal generalizado en la serie de CPUE (toneladas por día fuera de puerto) entre 1987 y 1999.

Table 3. Coefficients for the year effect obtained from generalized lineal model for CPUE (ton per days out of port), from 1987 to 1999.

\begin{tabular}{|cccc|}
\hline Año & Nominal & Estandarizado & $\begin{array}{c}\text { Error } \\
\text { estándar }\end{array}$ \\
\hline 1987 & 0,584 & 0,646 & 0,051 \\
1988 & 0,523 & 0,569 & 0,049 \\
1989 & 0,629 & 0,603 & 0,045 \\
1990 & 0,595 & 0,542 & 0,044 \\
1991 & 0,693 & 0,627 & 0,044 \\
1992 & 0,677 & 0,614 & 0,043 \\
1993 & 0,661 & 0,556 & 0,043 \\
1994 & 0,726 & 0,616 & 0,043 \\
1995 & 0,694 & 0,544 & 0,043 \\
1996 & 0,690 & 0,511 & 0,044 \\
1997 & 0,575 & 0,469 & 0,044 \\
1998 & 0,553 & 0,434 & 0,046 \\
1999 & 0,654 & 0,576 & 0,049 \\
\hline
\end{tabular}

Tabla 4. ANOVA del MLG ajustado a los datos de CPUE (toneladas por viaje con captura).

Table 4. ANOVA for MLG applied to the CPUE (ton per trips with capture).

\begin{tabular}{|ccrrrr|}
\hline Efecto & g.l. & $\begin{array}{c}\text { Suma } \\
\text { cuadrado }\end{array}$ & $\begin{array}{c}\text { Cuadrado } \\
\text { medio }\end{array}$ & $\begin{array}{c}\text { Valor } \\
\text { F }\end{array}$ & $\begin{array}{c}\text { Valor } \\
\mathbf{P}\end{array}$ \\
\hline Año & 12 & 87,55 & 7,30 & 129,05 & 0,000 \\
Estación & 3 & 101,54 & 33,85 & 598,67 & 0,000 \\
Estrato & 3 & 80,93 & 26,98 & 477,16 & 0,000 \\
Capacidad & & & & & \\
bodega & 1 & 170,05 & 170,05 & 3007,89 & 0,000 \\
$\begin{array}{c}\text { Año: } \\
\text { Estrato }\end{array}$ & 36 & 11,98 & 0,33 & 5,88 & 0,000 \\
Residuos & 3171 & 179,28 & 0,06 & & \\
\hline
\end{tabular}

realizada en el contexto de las características y dinámica de la pesquería. En este sentido, la incorporación de la Capacidad de Bodega (CB) en la modelación responde a la necesidad de contar con una variable continua que pueda dar cuenta de los cambios tecnológicos de la flota, ya que esta variable se ha incrementado para mejorar el rendimiento en captura de las embarcaciones que realizan la operación en zonas de caladeros más alejadas de los puertos base.

La principal limitante de esta investigación se encuentra en la falta de variables ambientales en la construcción de los modelos, las cuales no se incorporaron por carecer de esta información en la serie analizada, aunque se reconoce que deben ser incluidas a futuro para aumentar la validez de los modelos, ya que puede producir importantes sesgos en los análisis. Por ejemplo Punsly y Nakano (1992), encuentran que las tendencias en las tasas de captura estandarizadas a veces no están asociadas con las variaciones en la abundancia, ya que factores como nivel de oxígeno y la estructura térmica vertical producen considerables variaciones en la CPUE, no acordes con un cambio real en la abundancia. No obstante, una serie de CPUE estandarizada es de mejor utilidad que una serie nominal sin estandarizar y las variables incorporadas al ser significativas mejoran la representatividad de las series de CPUE como índice de abundancia relativa.

Otra fuente de incertidumbre en el análisis de las series de CPUE, se refiere a la caracterización de la flota por estratos, si bien la aplicación del ACP tiene la ventaja de agrupar las embarcaciones en 
Tabla 5. Coeficientes del efecto año obtenidos del modelo lineal generalizado en la serie de CPUE (toneladas por viaje con captura) entre 1987 y 1999.

Table 5. Coefficients for year effect obtained from generalized lineal model to CPUE (ton per trips with capture), from 1987 to 1999.

\begin{tabular}{|cccc|}
\hline Año & Nominal & Estandarizado & $\begin{array}{c}\text { Error } \\
\text { estándar }\end{array}$ \\
\hline 1987 & 0,662 & 0,759 & 0,060 \\
1988 & 0,634 & 0,718 & 0,058 \\
1989 & 0,722 & 0,731 & 0,053 \\
1990 & 0,724 & 0,688 & 0,052 \\
1991 & 0,828 & 0,778 & 0,052 \\
1992 & 0,944 & 0,876 & 0,051 \\
1993 & 1,015 & 0,879 & 0,051 \\
1994 & 1,078 & 0,921 & 0,050 \\
1995 & 1,065 & 0,872 & 0,050 \\
1996 & 1,119 & 0,845 & 0,052 \\
1997 & 0,980 & 0,815 & 0,052 \\
1998 & 1,060 & 0,865 & 0,054 \\
1999 & 1,098 & 0,934 & 0,058 \\
\hline
\end{tabular}

estratos homogéneos, se requiere una completa y actualizada fuente de información asociada a los cambios técnico-operacionales de las embarcaciones que operan de año en año, ya que es posible que producto de innovaciones técnico-operacionales, puedan existir embarcaciones que cambien de estrato mientras se desarrolla la pesquería. La situación anteriormente descrita puede ser corregida al efectuar una clasificación anual por estratos de las embarcaciones en actividad y lograr así una caracterización más precisa de las embarcaciones en toda la serie analizada.

Las diferentes tendencias observadas en ambas series de CPUE, pueden ser producto de la relación entre la dinámica del recurso y la flota, bajo una determinada unidad de esfuerzo de pesca. Para el caso de la serie de CPUE (ton/vcp) es posible que las tendencias observadas se encuentren relacionadas con períodos de cambios en la distribución espacial del recurso. Campos (1996), señala que la flota cerquera de jurel en la zona centro-sur ha debido variar su dinámica operativa entre 1994 y 1996, expandiendo su radio de acción, tanto en sentido norte-sur como hacia el oeste. De esta forma se verifica que los cambios de distribución anuales y estacionales del recurso afectan el régimen operacional de la flota. En consecuencia, los diferentes estratos que componen la flota pueden presentar cambios en su eficiencia asociados a la zona de pesca y la consecuente variabilidad en el esfuerzo de pesca aplicado sobre este recurso. Una situación común asociada a la interacción entre la flota y el recurso y su efecto sobre la CPUE, la constituye la agregación de embarcaciones de menor tamaño (270-400 m3 de CB) en sectores cercanos a los puertos base, bajo condiciones desfavorables de navegación (Campos, 1996).

Por otra parte, en el caso de encontrarse las zonas de pesca alejadas de los puertos base, son las embarcaciones con mayor autonomía las más eficientes en relación al esfuerzo de pesca. Situaciones como las descritas hacen evidente la necesidad de incorporar el efecto de la zona sobre las tasas de captura, para analizar si tiene un efecto significativo sobre la CPUE, en especial en un recurso con las características de movilidad y distribución del jurel. En esta investigación, la componente espacial no fue incorporada en la modelación debido a problemas de disponibilidad y cobertura temporal. Al igual que las variables ambientales, la incorporación de esta componente espacial mejoraría la representatividad de la serie de abundancia relativa, más aún en un recurso con las características del jurel. En este escenario, y considerando que la pesquería fue afectada por un sistema de vedas y regulaciones entre 1997 y 1999, la CPUE no constituye un buen indicador de la abundancia del recurso, aunque sí es posible conocer frente a qué factores son más sensibles las tasas de capturas de esta especie.

Entre los principales problemas asociados a la estandarización de la CPUE se encuentran las posibles migraciones hacia y desde el área cubierta por la información, la extensión geográfica de la población (Large, 1992) y la disponibilidad de información de variables que pueden influir sobre la CPUE (Hinton y Nakano, 1996). Al respecto, Hilborn y Walters (1992) señalan que para comprender la relación entre la CPUE y la abundancia relativa del recurso, se debe analizar la dinámica al interior del área, las características del área de pesca y del esfuerzo. Por otra parte, es necesario disponer de información complementaria de la flota, referida a variables asociadas a cambios en el sistema de pesca y presencia de equipo satelital (Allen y Punsly, 1984).

Los resultados obtenidos sugieren que en flotas altamente heterogéneas, la caracterización de las embarcaciones por medio de una análisis multivariado puede fortalecer las estimaciones de tasas de captura estandarizadas, facilitando la com- 
prensión de la interacción entre la flota y el recurso. La posibilidad de emplear estas series en los modelos indirectos edad-estructurados no es posible, ya que no se cuenta con todas las variables necesarias como serían las de tipo espacial y ambiental. Por lo tanto, sólo deben ser utilizadas para evaluar los principales factores que están asociados a la CPUE. Además, no es posible esperar que las tendencias en los índices de abundancia estandarizados hayan aislado por completo el efecto de las diferentes variables que pueden afectar la CPUE. No obstante, son mejores que un índice no estandarizado, ya que permiten indagar en las causas asociadas de variabilidad de los índices de abundancia relativa y/o disponibilidad en los recursos sometidos a una fuerte presión de pesca.

\section{AGRADECIMIENTOS}

El primer autor agradece las facilidades otorgadas por el Instituto de Investigación Pesquera (INPESCA) para desarrollar este estudio en el ámbito de la Unidad de Investigación presentada en el programa de Magíster en Ciencias con Mención en Pesquerías de la Universidad de Concepción. Los autores agradecen las sugerencias y comentarios de A. Sepúlveda.

\section{REFERENCIAS}

Allen, R. y R. Punsly. 1984. Catch rates as indices of abundance of yellowfin tuna, Thunnus albacares, in the eastern Pacific Ocean. Inter. Amer. Trop. Tuna Comm. Bull., 18(4): 301-379.

Böhm, M.G., M.A. Barbieri, G. Rosson, E. Yáñez, V. Catasti, A. González, L. Cubillos, A. Sepúlveda, A. Hernández y P. Sobarzo. 1997. Análisis de la captura y del esfuerzo de pesca en la pesquería de jurel de la zona centro-sur. Pre-Informe final FIP 96-18, 81 pp.

Campos, P. 1996. Análisis de las zonas de pesca y capturas de la flota industrial de cerco de la VIII Región en escalas espacio-temporales de mediano plazo. Tesis de Magíster en Oceanografía, Universidad de Concepción, 122 pp.

Chambers, J.M. y T.J. Hastie. 1993. Statistical Models in S. Chapman and Hall, New York, 608 pp.
Goñi, R., F. Álvarez y S. Adlerstein. 1999. Application of generalized linear modeling to catch rate analysis of Western Mediterranean fisheries: the Castellón trawl fleet as a case a study. Fish. Res., 42: 291-302.

Grechina, A. 1992. Historia de investigaciones y aspectos básicos de la ecología del jurel (Trachurus symmetricus murphyi (Nichols) en alta mar del Pacífico Sur. Doc. Téc. Inst. Invest. Pesq, (INPESCA), Talcahuano, 1(2): 1-47.

Hernández, A. y A. Sepúlveda. 1998. Análisis del esfuerzo y rendimiento de pesca aplicado al recurso jurel en la zona centro-sur de Chile entre 1987 y 1995. En: D. Arcos (ed.). Biología y ecología del jurel en aguas chilenas. Instituto de Investigación Pesquera, Talcahuano, $216 \mathrm{pp}$.

Hilborn, R. y C. Walters. 1992. Quantitative fisheries stock assessment. Chapman and Hall, New York, $570 \mathrm{pp}$.

Hinton, M.G. y H. Nakano. 1996. Standardizing catch and effort statistics using physiological, ecological, or behavioral constraints and environmental data, with an application to blue marlin (Makaira nigricans) catch and effort data from Japanese longline fisheries in the pacific. Inter. Amer. Trop. Tuna Comm. Bull., 21(4): 171-197.

Kimura, D.K. 1981. Standardized measures of relative abundance based on modeling log (c.p.u.e), and their application to Pacific ocean perch (Sebastes alutus). J. Cons. int. Explor. Mer., 39: 211-218.

Kimura, D.K. y H.H. Zenger 1997. Standardizing sablefish (Anoplopoma fimbria) long-line survey abundance indices by modeling the log-ratio of paired comparative fishing cpues. ICES, J. Mar. Sci., 54: 48-59.

Large, P.A. 1992. Use of a multiplicative model to estimate relative abundance from commercial CPUE data. ICES. J. Mar. Sci., 49: 253-261.

Mc Cullagh, P. y J.A. Nelder. 1989. Generalized linear models. Chapman and Hall, New York, 511 pp.

Pease, B. 1999. A spatially oriented analysis of estuaries and their associated commercial fisheries in New South Wales, Australia. Fish. Res., 42: 6786.

Punsly, R.G. y R. Deriso. 1991. Estimation of relative annual abundance of yellowfin tuna, Thunnus 
albacares, by age groups and regions within the eastern pacific Ocean. Inter. Amer. Trop. Tuna Comm. Bull., 20: 97-131.

Punsly, R.G. y H. Nakano. 1992. Analysis of variance and standardization of longline hook rates of biyege (Thunnus obesus) and yellowfin (Thunnus albacares) in the eastern Pacific Ocean during 1975-1987. Inter. Amer. Trop. Tuna Comm. Bull., 20: $165-184$.

Punt, A.E., T.I. Walker, B.L. Taylor y F. Pribac. 2000. Standarization of catch and effort data in a spatially-structured shark fishery. Fish. Res., 45: 129-145.

Quinn, T.J. y R.B. Deriso. 1999. Quantitaive fish dymamics. Oxford University Press, New York, $542 \mathrm{pp}$.

Sainsbury, K., T. Polacheck, N. Klaer, J. Gunn, R. Campbell, W. Hearn, T. Davis, A. Betlehem, A. Preece y A. Cowling. 1999. Recent developments and methods in the southern bluefin tuna fishery assesments. En: G.T. DiNardo (ed.). Proceedings of the Second International Pacific Swordfish Symposium. NOAA-TM-NMFS-SWFSC-263 June, pp. 135-158.

Serra, R. 1991. Important life history aspects of the Chilean jack mackerel, Trachurus symmetricus murphyi. Invest. Pesq., Chile, 36: 67-83.
Serra, R., G. Böhm y M. Donoso. 1998a. Situación de las pesquerías pelágicas zona centro-sur. Programa "Seguimiento del estado de situación de las principales pesquerías nacionales". Informe Final. Instituto de Fomento Pesquero, Chile, $61 \mathrm{pp}$.

Serra, R., A. Zuleta, H. Pool y G. Böhm. 1998b. Bases biológicas para prevenir la sobreexplotación en el recurso jurel. Informes Técnicos, Fondo de Investigación Pesquera, FIP-IT/96-16, 139 pp.

Shimada, B.M. y M.B. Shaeffer. 1956. A study of changes in fishing effort, abundance, and yield for yellowfin and skipjack tuna in the eastern tropical Pacific Ocean. Inter. Amer. Trop. Tuna Comm. Bull., 1(7): 351-421.

Simpfendorfer, C.A., K. Donohue y G.H. Norm. 2000. Stock assessment and risk analysis for the whiskery shark (Furgaleus macki (Whitly)) in south-western Australia. Fish. Res., 47: 1-17.

Subsecretaría de Pesca (SUBPESCA). 1999. Cuota global de captura para la pesquería del recurso jurel, año 2000. Subsecretaría de Pesca, Informe Técnico, 64, 32 pp.

Yáñez, E., F. Espíndola, P. Fréon y M.A. Barbieri. 1999. Estandarización de tasas de captura de pesquerías pelágicas de la zona norte de Chile durante 1987-92. Invest. Mar., Valparaíso, 27: 53-63. 